nearly independent of the amount of free bromine in this region of concentration.

Runs with free iodine instead of bromine or chlorine gave no reaction.

An experiment with symmetric tetra. bromethane and free chlorine gave a marked reaction. A yellow, viscous oil was isolated.

A rough test with radioactive free chlorine and inactive hexabromoethane showed an uptake of chlorine activity in the organic phase, later falling off again.

The curves, Fig. 2, show that the organic bound activities fall to at least $2 \%$ of the original activities. Setting aside isotope effect, this may indicate a polymer consisting of some system of condensed carbon rings.

Further investigations are in progress.

1. Seely, L. B., Jr. and Willard, J. E. J. Am. Chem. Soc. 69 (1947) 2061.

2. Mouneyrat, X. Bull. soc. chim. 19 (1898) 177.

3. Dickinson, R. G. and Leermakers, J. A. J. Am. Chem. Soc. 54 (1932) 3853.

Received November 6, 1957.

\section{The Precipitation of Neutral Polysaccharides by Cationic Detergents}

HANS PALMSTIERNA, J. E. SCOTT* and 9. GARDELL

\section{Depts. of Chemistry and Bacteriology}

Karolinska institutet, Stockholm, Sweden

$D_{\mathrm{p}}$ uring an investigation on the charged polysaccharide luteic acid, described by Raistrick and collaborators in $1931^{1}$, the method of Scott was used as one step in the purification of it. It has been shown 2 that it is possible to use detergents for the precipitation and separation of mixtures of charged polysaccharides (and desoxyribonucleic acid). For obvious reasons neutral polysaccharides cannot be precipitated directly by the use of detergents such as cetylpyridinium chloride (C.P.C.) or cetyltrimethylammonium bromide (C.T.A.B.). If, however, the neutral polysaccharide is

* Present address. Institute of Pathology and Tropical Medicine, R. A. F. Halton Bucks, Fingland. coupled to borate and C.P.C. is added, complete precipitation can be achieved. Since the work in this paper was carried out a short communication has appeared also recording this fact ${ }^{3}$. The precipitates are soluble in salt solutions, dilute scids and glycerol and galactose solutions.

To eliminate the effect of buffer salts on the precipitate, cetyl-trimethyl ammonium borate (C.T.A. Borate) was prepared and used as the precipitant. The $\mathrm{pH}$ dependance of the reaction was marked, as was observed by Barker et al. ${ }^{\mathrm{s}}$ Rat liver glycogen was not precipitated by C.T.A. borate, which had a pH of 8.5 , but the addition of a fow drops of ammonia resulted in good precipitation. Yeast mannan and ivory nut mannan were precipitated immediately without the addition of ammonia. Inulin, dextran and starch were not precipitated even in the presence of ammonia, but only on adding an appreciable concentration of potassium hydroxide. This latter type of precipitation in potassium hydroxide also took place in the absence of borate, and although the precipitates were soluble in selt solutions they were resistant to solvent action by glycerol or galactose.

It is therefore clear that there are two possibilities of precipitating neutral polysaccharides with amphipathic cations, due to the acquiring of polyanionic properties:

(1) by formation of borate complexes, and

(2) by the ionisation of hydroxyl groups at high $\mathrm{pH}$.

The possibility of salt fractionation, such as is available with acidic polysaccharides ${ }^{2}$, coupled with the differential solubilities of the precipitates in glycerol and galactose solutions, suggest that this reaction has great potential practical value. The follow. ing experiment describes a simple test of its utility (see also Barker et al. ${ }^{8}$ ).

The test system used was glycogen from Escherichia coli B alone or in combination with luteic acid. It was possible to separate these polysaccharides by precipitating the luteic acid directly with C.P.C. The glycogen was then precipitated by making the solution $0.01 \mathrm{M}$ with regard to borate, adjusting the $\mathrm{pH}$ to 9.2 with $\mathrm{KOH}$ and finally adding C.P.C. to complete precipitation. This last operation should be finished within an hour, since the solution may otherwise be discoloured due to slight decomposition of C.P.C. in alkaline solutions. The supernatant contrined hardly detectable amounts of polysaccharide. 
The glycogen was easily dissociated from the borate-C.P.C. complex by lowering the pH to neutrality. This was followed by a dialysis against tap water. The luteic acid was separated from the C.P.C. by the following procedure ${ }^{2}$.

The complex was dissolved in a solvent consisting of methanol $20 \% \mathrm{v} / \mathrm{v}$, saturated sodium chloride $30 \% \mathrm{v} / \mathrm{v}$ and water to $100 \%$. This solution was treated with Lloyds reagent and the disappearance of the C.P.C. was followed spectrophotometrically.

It should be pointed out the method cannot be used when large amounts of protein contaminate the polysaccharide solution, because the proteins tend to form highly viscous cakes with detergents, thus enclosing the polysaccharides.

In microbiological work extracellular polysaccharides can in many instances be isolated by the above methods if the salt concentration of the medium is not too high. When the salt content is high it is advisable to dilute the medium or to dialyse it before precipitation with C.P.C.

1. Raistrick, H. et al. Phil. Trans. Roy. Soc. London, Ser. B 220 (1931).

2. Scott, J. E. Chemistry \& Industry 1955 1568; Biochem. J. 62 (1956) 31P.

3. Barker, S. A. et al. Chemistry \& Industry 1957330.

Received November 13, 1957.

\section{Isolation of Oestradiol-17 $\alpha$ from Bovine Meconium \\ WEIERT VELLE}

\section{Department of Sexual Physiology and Sexual Pathology, Veterinary College of Norway, Oslo, Norway}

$I^{\mathrm{n}}$ nvestigations on oestrogens in the new. Iborn calf have shown that significant amounts of oestrone and oestradiol-17a are excreted with the urine during the first days of life ${ }^{1}$. These observations led to investigations on the oestrogen contents of meconium in this species.

In this preliminary communication results are presented which strongly indicate the presence of significant quantities of oestradiol-17a in meconium from calves.
Experimental. Meconium was obtained from normal, healthy, newborn calves of both sexes and the analyses were undertaken immediately.

Reagents were of the same grade and manu. facture as those used in a previous investigation ${ }^{2}$. The material was examined for the contents of both free and conjugated oestrogens:

a. For determination of free oestrogens specimens of meconium were suspended in distilled water and disintegrated in an "Ato-Mix" blender. The suspensions were then extracted repestedly with ether. The combined ether extracts were processed as described in the following.

b. For determination of conjugated oestrogens the water phase from a. was freed from ether by evaporation, and concentrated $\mathrm{HCl}$ added to a final concentration of $10 \%$. Fitted with reflux condenser, the flask was kept at $100^{\circ} \mathrm{C}$ for $1 \mathrm{~h}$. After cooling ether extraction was performed as before. The combined ether extracts were analysed according to the following procedures:

1. The chemical method for determination of urinary oestrogens, described by Brown ${ }^{3}$ was applied with minor modifications ${ }^{2}$. This method is based on the chromatographic sepa. ration of the 3-methyl ethers of the oestrogens, using standardized alumina as adsorbent.

2. Chromatographic separation of the un. altered oestrogens were performed according to the method of Stimmel as modified by Kakusjkina and Orlova ${ }^{5}$.

3. The cis-trans test for the 17-hydroxy group of oestradiol was performed according to Kägi and Miescher ${ }^{6}$, omitting the addition of bromine.

4. Absorption spectra of the isolated fractions were examined from $400-600 \mathrm{~m} \mu$ using the Kober reagent described by Bauld ?, and from $240-500 \mathrm{~m} \mu$ using fuming sulphuric acid according to Axelrod ${ }^{8}$. The registered spectra were compared with those of pure compounds. All readings were made in a Beckman Model DU spectrophotometer supplied with photo. multiplier.

Results and discussion. Using the modi. fied Brown procedure, a strongly reacting Kober chromogen was found present in the oestradiol fractions from both a. and b., indicating that the substance is partly excreted in free and partly in conjugated form. Considerably greater quantities were observed in the "conjugated" fractions.

In the oestriol and oestrone fractions no trace of Kober chromogen could be detected. 